MAGDA MARIA MAIA

\title{
Teste cardiopulmonar de exercício em pacientes com fibromialgia juvenil
}

Tese apresentada à Faculdade de Medicina da Universidade de São Paulo para obtenção do título de Doutor em Ciências

Programa de Ciências Médicas

Área de Concentração: Processos Imunes e Infecciosos Orientador: Prof. Dr. Clovis Artur Almeida da Silva

São Paulo 2016 
Dados Internacionais de Catalogação na Publicação (CIP)

Preparada pela Biblioteca da

Faculdade de Medicina da Universidade de São Paulo

Creprodução autorizada pelo autor

Maia, Magda Maria

Teste cardiopulmonar de exercício em pacientes com fibromialgia juvenil / Magda Maria Maia. -- São Paulo, 2016.

Tese(doutorado)--Faculdade de Medicina da Universidade de São Paulo.

Programa de Ciências Médicas. Área de Concentração: Processos Imunes e Infecciosos.

Orientador: Clovis Artur Almeida da Silva.

Descritores: 1.Fibromialgia 2.Criança 3.Adolescente 4.Sistema nervoso autônomo 5.Teste de esforço 6.Tolerância ao exercício 7.Reserva cronotrópica 8.Disfunção autonômica 9.Qualidade de vida 10.Estudos tranversais

USP/FM/DBD-422/16 


\section{Dedicatória}


Aos meus pais Orides Maia (in memoriam) e Petrina pelos princípios e valores a mim ensinados, por estarem sempre presentes, mesmo estando distante, por oferecer amor e carinho incondicional aos filhos e netos.

Aos meus filhos Luciana e Eduardo, por estarem ao meu lado e me apoiarem nesta jornada, por serem a motivação e o amor da minha vida. 
Agradecimentos 
Ao meu orientador, Clovis Artur Almeida da Silva, professor e médico dedicado, "ser humano impar", exemplo para seus alunos, por contar com o seu entusiasmo contagiante, com a sua alegria e o seu incentivo em todos os momentos. Agradeço, ainda, por sua generosidade, pela confiança e sua a disponibilidade ao longo desses anos, que foram essenciais para a continuidade e finalização deste trabalho;

Aos médicos Adriana Maluf Elias Sallum, Lúcia Maria de Arruda Campos, Nádia Emi Aikawa, do ambulatório de reumatologia pediátrica do Instituto da criança, Claudio Len, Maria Teresa A. Terreri, Melissa Fraga, Vanessa Bugni, Daniela Petri do ambulatório de reumatologia pediátrica da UNIFESP e por seu apoio na avaliação dos pacientes e ensinamentos sobre fibromialgia juvenil, e todos os residentes, por terem compartilhado comigo o seu aprendizado e a Dra. Cássia M. Barbosa Passarelli e ao Dr. Sérgio A. Bastos Sarrubbo, por apoiarem a participação do Hospital Infantil Darcy Vargas neste estudo;

À Dra. Ana Lúcia de Sá Pinto, ao Hamilton Roschel, ao Bruno Gualano pela orientação na elaboração deste projeto e por todos os ensinamentos sobre o teste de ergoespirométrico;

Ao Luiz Perandini, Danilo Prado, Thalita Dassouki, Lilian Takayama e a toda equipe do LACRE e LIN 17 pela colaboração e apoio aos pacientes;

À Mariza Yoshikawa e á Valéria Vilhena, pelo apoio nas pesquisas bibliográficas, sempre disponíveis e atenciosas e ao Quintino pela orientação sobre os descritores e presteza na elaboração da ficha catalofráfica;

À minha filha Luciana Magalhães, por me apoiar e auxiliar na elaboração do banco de dados e tabelas; 
Aos meus amigos Marcela Moreira, Luciana Pacheco e Luiz Correia, por terem convidado parentes e amigos para participar deste projeto;

Aos meus amigos e parceiros de vida profissional, Marcos Thomazin e Edi Toma, por me incentivar, ouvir e aconselhar;

À Dra. Carmela Maggiuzzo Grindler e à Dra. Leonete Guimaraes Ferreira, pessoas importantes, que me proporcionaram a oportunidade de crescimento profissional, pela compreensão e apoio indispensáveis para conciliar a minha jornada de trabalho e a elaboração desta tese;

À todos os jovens adolescentes e familiares, por participarem deste estudo. 
“Aqueles que passam por nós, não vão sós, não nos deixam sós. Deixam um pouco de si, levam um pouco de nós."

Antoine de Saint - Exupéry 
Esta tese está de acordo com as seguintes normas, em vigor no momento desta publicação:

Referências: adaptado de International Committee of Medical Journals Editors (Vancouver).

Universidade de São Paulo. Faculdade de Medicina. Divisão de Biblioteca e Documentação. Guia de apresentação de teses. Elaborado por Anneliese Carneiro da Cunha, Maria Julia de A. L. Freddi, Maria F. Crestana, Marinalva de Souza Aragão, Suely Campos Cardoso, Valéria Vilhena. 3a ed. São Paulo: Divisão de Biblioteca e Documentação; 2011.

Abreviaturas dos títulos dos periódicos de acordo com List of Journals Indexed in Index Medicus. 


\section{SUMÁRIO}

Lista de abreviaturas e siglas

Lista de símbolos

Listas de figuras

Listas de tabelas

Resumo

Abstract

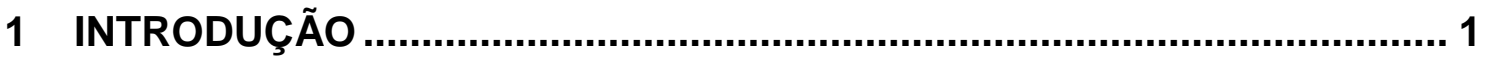

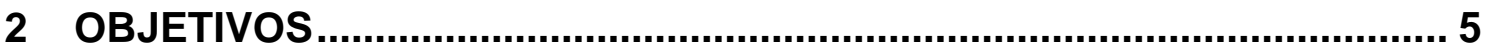

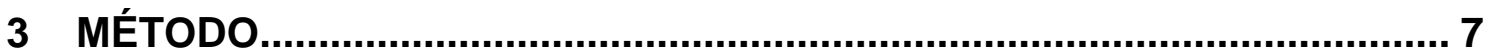

3.1 Desenho do estudo e participantes ....................................... 8

3.1.1 Critérios de inclusão e exclusão dos pacientes ..................... 9

3.1.2 Critérios de inclusão e exclusão indivíduos saudáveis ............. 9

3.1.3 Critérios de exclusão relacionados à prática de atividade física............................................................................... 10

3.1.4 Aspectos éticos ................................................ 10

3.2 Procedimentos do estudo .................................................... 11

3.2.1 Dados demográficos .............................................. 11

3.2.2 Avaliação inicial da capacidade funcional e qualidade de vida relacionada à saúde (QVRS), índice de dor e critérios de hipermobilidade articular ........................................ 12

3.2.3 Teste cardiopulmonar de exercício ............................... 13

3.3 Análise estatística............................................................. 16

4 RESULTADOS.................................................................................. 17

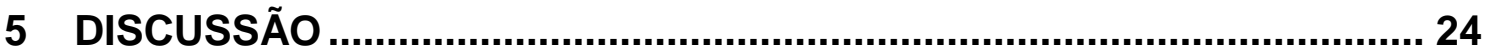

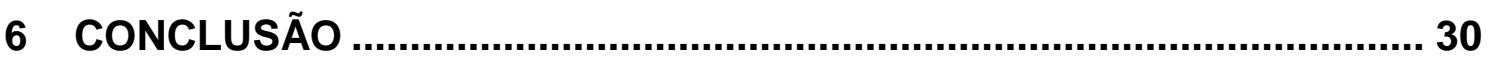

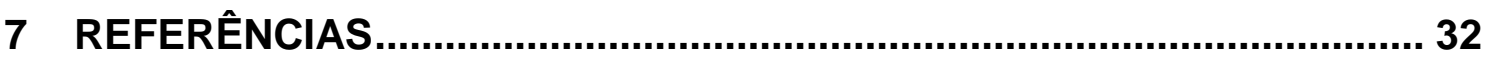




\section{LISTA DE ABREVIATURAS E SIGLAS}

AIJ

CHAC

FMJ

FC

IC

IMC

LA

LESJ

PCR

Peds QL

QVRS

$\mathrm{RC}$

FCR

RTR

$\mathrm{VO}_{2}$

TCPE
Artrite idiopática juvenil

Childhood Health Assessment Questionnaire

Síndrome da fibromialgia juvenil

Frequência cardíaca

Incompetência cronotrópica

Índice de massa corporal

Limiar ventilatório anaeróbio ou limiar anaeróbio

Lúpus eritematoso sistêmico

Ponto de compensação respiratória

Pediatric Quality of Life Inventory

Qualidade de vida relacionada à saúde

Reserva cronotrópica

Frequência cardíaca de recuperação

Razão de troca respiratória

Consumo de oxigênio

Teste cardiopulmonar de exercício 


\section{LISTA DE SÍMBOLOS}

$\mathrm{Kg}$

$\mathrm{cm}$

$\mathrm{Kg} / \mathrm{m}^{2}$

$\mathrm{ml} / \mathrm{kg} / \mathrm{min}$

$\mathrm{mph}$

W (potência)

STPD

BTPS quilograma

centímetro

quilograma por metro cúbico

mililitro por quilograma por minuto

milhas por hora

watt (joule/segundo, medida de energia/trabalho)

standard temperature pressure and dry

body temperature pressure saturated 


\section{LISTA DE FIGURAS}

Figura 1 - Fluxograma da pesquisa

\section{LISTA DE TABELAS}

Tabela 1 - Dados demográficos e estágio puberal de Tanner em pacientes com fibromialgia juvenil primária (FMJP) e controles saudáveis ... 18

Tabela 2 - Estatística descritiva da avaliação clinica e funcional dos pacientes com fibromialgia juvenil primária (FMJP)

Tabela 3 - Dados qualidade de vida relacionada à saúde (QVRS) em pacientes com fibromialgia juvenil primária (FMJP) e controles saudáveis

Tabela 4 - Dados cardiovasculares mensurados durante e após exercício de esforço máximo em pacientes com Fibromialgia juvenil primária (FMJP) e controles saudáveis

Tabela 5 - Coeficiente de correlação de Pearson's para os parâmetros de QVRS, CHAQ, escore de dor e dados cardiorrespiratórios mensurados nos pacientes com fibromialgia juvenil primária 


\section{RESUMO}

Maia MM. Teste cardiopulmonar de exercício em pacientes com fibromialgia juvenil [tese]. São Paulo: Faculdade de Medicina, Universidade de São Paulo; 2016.

Introdução: A disfunção do sistema nervoso autônomo (disfunção autonômica ou dissautonomia) tem sido associada à fisiopatologia da fibromialgia em pacientes adultos. A modulação cardíaca em resposta ao exercício foi demonstrada em uma série de estudos em adultos com fibromialgia que evidenciaram reduzida capacidade aeróbia, assim como o comprometimento autonômico cardíaco e incompetência cronotrópica, que é a incapacidade de aumentar a frequência cardíaca concomitante ao aumento da intensidade do exercício. No entanto, a capacidade aeróbica e a disautonomia, definidas a partir da avaliação dos parâmetros do teste de exercício cardiopulmonar, não foram estudadas em pacientes adolescentes com síndrome da fibromialgia (FMJ). Objetivo: Avaliar os parâmetros do teste de exercício cardiopulmonar em pacientes com FMJ e controles saudáveis e as possíveis correlações entre estes parâmetros e a qualidade de vida relacionada à saúde (QVRS), capacidade funcional e dor nos pacientes FMJ. Métodos: Estudo transversal multicêntrico incluindo 25 pacientes com FMJ e 25 controles saudáveis. Ambos os grupos participavam somente das aulas de educação física na escola. O teste de exercício cardiopulmonar de esforço em esteira permitiu avaliar a resposta cardiorrespiratória durante 0 exercício. A resposta cronotrópica foi avaliada pela medida da reserva cronotrópica. Foram avaliados dor, capacidade funcional e QVRS. Resultados: A mediana da idade atual foi similar nos pacientes com FMJ e controles saudáveis (15 vs. 15 anos, $p=0,890)$, assim como o índice de massa corporal $(p=0,332)$, gênero feminino $(p=1,000)$ e estágios de Tanner $(p=0,822)$. A mediana dos parâmetros da QVRS (escore total de saúde física e saúde psicossocial) foi significativamente menor nos pacientes com FMJ versus controles, de acordo com o autorrelato dos pacientes e de seus pais $(p<0,001)$. A mediana do pico FC [181 (150-198) vs. 197 (181-202) bpm, $\mathrm{p}<0,001]$, da reserva cronotrópica (RC) [84 (53-98) vs. 99 $(84-103) \%, p<0,001]$ e da $\mathrm{FC}$ de repouso à $\mathrm{FC}$ de pico [96 (65-181) vs. 127 (61-185) bpm, $\mathrm{p}=0,010]$ foram significantemente menores nos pacientes com FMJ quando comparados aos controles saudáveis. A mediana do $\triangle \mathrm{FCR} 1$ [15 (3-39) vs. 35 (9-52) bpm, $p<0,001], \Delta \mathrm{FCR} 2$ [37 (20-57) vs. 51 (32-94) bpm, $\mathrm{p}<0,001]$, VO2 de pico [32.34 (24.24-39.65) vs. 36.4 (28.56-52.71) $\mathrm{ml} / \mathrm{kg} / \mathrm{min}, \mathrm{p}=0,005]$, velocidade máxima [5 (4-6.3) vs. $5.9(4.0-6.3) \mathrm{mph}, \mathrm{p}=0,001]$, tempo de exaustão [11.5 (8.5-14.5) vs. $14(11-18)$ minutos, $p<0,001]$ e capacidade de trabalho [3.37 (2.04-5.6) vs. 3.89 (2.91-6.55) W/kg, $\mathrm{p}=0,006$ ] foram significativamente menores nos pacientes com FMJ quando comparados aos controles. A frequência da incompetência cronotrópica $(\leq 80 \%)$ foi significativamente maior nos pacientes com FMJ versus controles $(p=0,0006)$. Conclusões: Este estudo identificou incompetência cronotrópica e recuperação atenuada da $\mathrm{FC}$ em pacientes com FMJ, indicando disfunção autonômica.

Descritores: fibromialgia; criança; adolescente; sistema nervoso autônomo; teste de esforço; tolerância ao exercício; reserva cronotópica; disfunção autonômica; qualidade de vida. 


\begin{abstract}
Maia MM. Cardiopulmonary Exercise Test in patients with Juvenile fibromyalgia syndrome [thesis]. São Paulo: "Faculdade de Medicina, Universidade de São Paulo" 2016.
\end{abstract}

Introdução: Autonomic nervous system dysfunction (also named autonomic disturbance or dysautonomia) has been linked to physiopathology of adult patients with fibromyalgia. Cardiac modulation in response to exercise in case series of adult fibromyalgia revealed reduced aerobic capacity, as well as cardiac autonomic impairment and chronotropic incompetence, which is the inability to increase heart rate with an increase in exercise intensity. However, to our knowledge treadmill cardiorespiratory test and to assess aerobic capacity and dysautonomia has not been studied in adolescents with JFM patients. Objective: To assess cardiorespiratory exercise test parameters in Juvenile fibromyalgia syndrome (JFM) patients and healthy controls and possible correlations between these parameters and health-related quality of life (HRQL), functional ability and pain in JFM patients. Methods: A multicenter cross-sectional study included 25 JFM patients and 25 healthy controls. Both groups were engaged only in the physical education classes in school. A treadmill graded cardiorespiratory test was performed and heart-rate (HR) response during exercise was evaluated by the chronotropic reserve (CR). Pain, functional ability and HRQL were assessed. Results: The median current age was similar in JFM and controls (15 vs. 15years, $p=0.890$ ), as well as body mass index $(p=0.332)$, female gender $(p=1.000)$ and Tanner stages $(p=0.822)$. The medians of HRQL parameters (total score/physical health/psychosocial health) were significantly lower in JFM versus controls according to patient and parent self-reports $(p<0.001)$. The median of peak HR [181 $(150-198)$ vs. 197 (181-202) bpm, $p<0.001]$, chronotropic reserve [84 (53-98) vs. 99 (84-103)\%, $\mathrm{p}<0.001$ ] and resting to peak [96 (65-181) vs. 127 (61-185) bpm, $\mathrm{p}=0.010$ ] were significantly lower in JFM compared to controls. The median of $\triangle \mathrm{HRR} 1$ [15 (3-39) vs. 35 (9-52) bpm, $p<0.001$ ], $\Delta \mathrm{HRR} 2$ [37 (20-57) vs. 51 (32-94) bpm, $p<0.001$ ], peak $\mathrm{VO}^{2}$ [32.34 (24.24-39.65) vs. 36.4 (28.56-52.71) $\left.\mathrm{ml} / \mathrm{kg} / \mathrm{min}, \mathrm{p}=0.005\right]$; peak speed [5 (4-6.3) vs. $5.9(4.0-6.3) \mathrm{km} / \mathrm{h}, \mathrm{p}=0.001$ ], time to exhaustion [11.5 $(8.5-14.5)$ vs. $14(11-18)$ minutes, $p<0.001]$ and working capacity on power [3.37 (2.04-5.6) vs. $3.89(2.91-6.55) \mathrm{W} / \mathrm{kg}, \mathrm{p}=0.006$ ] were significantly lower in JFM compared to controls. The frequency of chronotropic incompetence $(\leq 80 \%)$ was significantly higher in JFM versus controls $(p=0.0006)$. Conclusions: This study identified chronotropic incompetence and delayed HR recovery in JFM patients, indicating autonomic dysfunction.

Descriptors: fibromyalgia; child; adolescent; autonomic nervous system; exercise test; exercise tolerance; chronotropic reserve; autonomic dysfunction; quality of life; cross-sectional studies. 
A fibromialgia (FM) é uma síndrome caracterizada por dor músculoesquelética crônica e difusa. A dor é considerada de origem central, decorrente de anormalidades na modulação dos processos dolorosos, e associada a uma série de sintomas sistêmicos, como fadiga, alteração do sono, cefaleia, dor abdominal, ansiedade, alteração do humor e dificuldades $\operatorname{cog}$ itivas ${ }^{1,2,3,4}$. Os sintomas da fibromialgia podem diferir em número, intensidade e frequência, sendo influenciados por estresses físico e psicológico ${ }^{5,6}$.

Estima-se que a fibromialgia juvenil (FMJ) afete 2,1-6,1\% das crianças e dos adolescentes, principalmente do sexo feminino ${ }^{7,8}$. Aproximadamente $20 \%$ dos casos de fibromialgia começam durante a infância ou na adolescência e podem persistir na vida adulta. Um extenso período decorre desde o início dos sintomas ao diagnóstico ${ }^{9}$.

A FMJ é associada com deficiências físicas para realizar diversas atividades diárias. A dor experimentada por esses pacientes é amplificada e o seu maior impacto está no comprometimento físico funcional, na condição geral de saúde, causando estresse emocional e sintomas de ansiedade e depressão. Além disto, pode acarretar impacto psicossocial e comprometimento da qualidade de vida relacionado à saúde (QVRS) $)^{10,11}$.

A etiologia e a patogênese da fibromialgia são desconhecidas. Atualmente, a sintomatologia da doença tem sido relacionada a possíveis disfunções do sistema nervoso autônomo. A disfunção do sistema nervoso autônomo (também chamada de distúrbio autonômico ou disautonomia) tem sido associada à fisiopatologia da fibromialgia em pacientes adultos ${ }^{12,13,14}$. 
A disautonomia em pacientes adultos com FM é caracterizada por hiperreatividade simpática, hiporreatividade ao estresse, hiperestimulação crônica dos receptores $\beta$-adrenégicos (e de outros receptores adrenérgico) e a desensibilização dos receptores ${ }^{15,16}$. Esta anormalidade pode fundamentar as características multissistêmicas da FM, incluindo fadiga crônica, intolerância ao calor, síncope, palpitação, desconforto torácico, distúrbios do sono e ansiedade $^{15}$. A disautonomia pode ser confirmada por testes de função autonômica cardíaca, tais como análise de variabilidade da frequência cardíaca (FC) e testes de estresse ${ }^{17,18}$.

Além disso, o sistema nervoso autônomo é importante na regulação do sistema cardiovascular em crianças e adultos, particularmente no controle da FC durante e após o exercício aeróbio gradual ${ }^{19}$. Durante o exercício, o aumento da FC é regulado por redução da atividade vagal, e aumento da atividade simpática, bem como por alterações de sensibilidade no nó sinoatrial, em resposta às catecolaminas. Em contraste, o aumento do tônus parassimpático no nó sinoatrial induz ao rápido declínio na $\mathrm{FC}^{20,21}$.

A resposta da FC ao teste de exercício progressivo fornece medidas válidas para mensurar a função autonômica cardíaca ${ }^{19}$. A incompetência cronotrópica (IC) é definida como a inabilidade de alcançar pelo menos $80 \%$ da reserva cronotrópica $(\mathrm{RC})$, determinada pela diferença entre a FC máxima prevista e a $\mathrm{FC}$ em repouso ${ }^{20,21}$. A recuperação da $\mathrm{FC}$ reflete a rápida reativação vagal e está correlacionada com a capacidade funcional. Este parâmetro também é considerado uma medida da função autonômica cardíaca em adultos $^{22,23}$ e em crianças $^{24}$. 
Tanto a IC quanto a recuperação anormal da FC são variáveis do teste de exercício e foram consideradas preditores de eventos cardíacos e de mortalidade em adultos com doença cardiovascular ${ }^{22,23}$, assim como em crianças e adolescentes com doenças cardíacas congênitas ${ }^{24}$. Além disto, estudos envolvendo pacientes adultos com fibromialgia submetidos ao teste de exercício mostraram reduzida capacidade aeróbia, ${ }^{12,20}$ assim como comprometimento autonômico cardíaco, incompetência autonômica e recuperação da FC atenuada ao final do teste ${ }^{12}$.

O teste cardiopulmonar de exercício vem sendo cada vez mais usado para identificação da intolerância ao exercício e da capacidade cardiopulmonar em crianças e adolescentes ${ }^{25,26,27,28}$. A capacidade funcional e as disfunções autonômicas foram estudadas em pacientes adultos com FM submetidos ao teste de exercício cardiopulmonar. No entanto, testes de exercício cardiopulmonar em adolescentes com FMJ no momento do diagnóstico ainda não foram estudados nesta população, particularmente avaliando a capacidade aeróbica e a função autonômica cardíaca. 
2 OBJETIVOS 
1. Avaliar os parâmetros do teste cardiopulmonar de exercício em pacientes com FMJ e controles saudáveis.

2. Identificar possíveis correlações entre parâmetros do teste cardiopulmonar de exercício, qualidade de vida relacionada à saúde, dor e capacidade funcional nos pacientes com FMJ. 


\subsection{Desenho do estudo e participantes}

Este estudo de caso-controle transversal foi conduzido no período de março de 2011 a fevereiro de 2015. Durante este período, foram recrutados 63 casos novos de pacientes diagnosticados com FMJ em quatro serviços terciários de reumatologia pediátrica na cidade de São Paulo, Brasil: Instituto da Criança do Hospital das Clínicas da Faculdade de Medicina da Universidade de São Paulo (ICr-HC-FMUSP), Disciplina de Reumatologia do HC-FMUSP, Universidade Federal de São Paulo e Hospital Infantil Darcy Vargas.

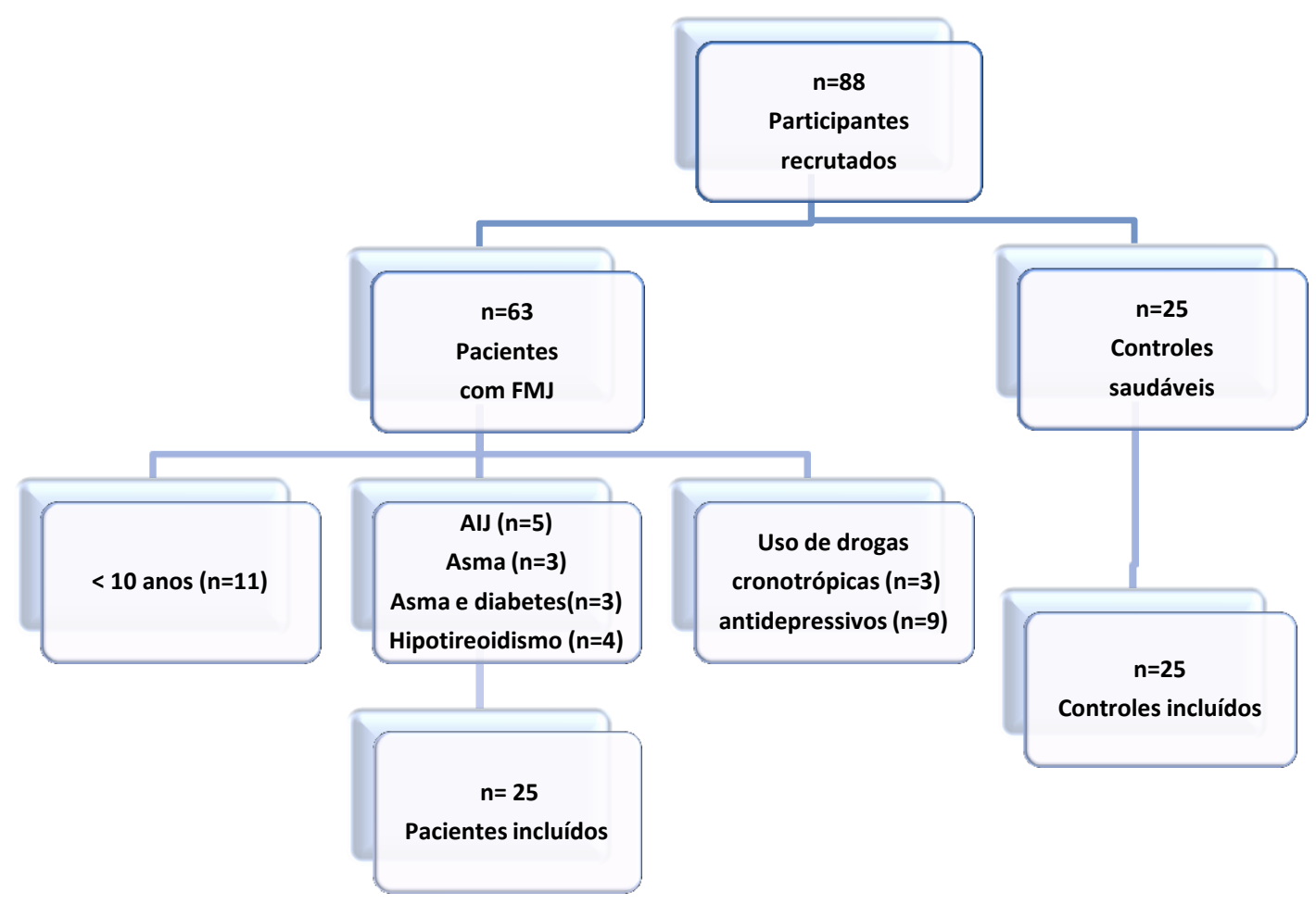

Figura 1 - Fluxograma da pesquisa 


\subsubsection{Critérios de inclusão e exclusão dos pacientes}

Foram incluídos adolescentes com FMJ, menores de 18 anos, diagnosticados de acordo com os critérios do American College of Rheumatology $2010^{29}$

Foram excluídos os pacientes com comprometimento cardiopulmonar (miocardite, pericardite, arritmia, hipertensão arterial, insuficiência cardíaca e asma); uso de tabaco; uso de drogas cronotrópica, antidepressiva e antihipertensiva; doença crônica pediátrica [hipotireoidismo, hipertireoidismo, lúpus eritematoso sistêmico juvenil (LESJ), artrite idiopática juvenil (AIJ), asma e diabetes tipo1] e os pacientes que se recusaram a participar deste estudo. Trinta e oito pacientes com FMJ foram excluídos devido: idade menor do que 10 anos $(n=11)$; AIJ $(n=5)$; uso de drogas cronotrópicas $(n=3)$; hipotireoidismo $(n=4)$; asma $(n=3)$; diabetes e asma $(n=3)$; e recusa em participar deste estudo por motivos particulares $(n=9)$. Portanto, 25 pacientes com FMJ foram selecionados para este estudo no momento do diagnóstico. No início do estudo, os pacientes faziam uso de medicamentos analgésicos 22/25 (88\%) e anti-inflamatórios não hormonais 3/25 (12\%). Nenhum paciente com FMJ foi submetido à terapia cognitivo-comportamental ou a uso de droga antidepressiva.

\subsubsection{Critérios de inclusão e exclusão indivíduos saudáveis}

No grupo controle, foram incluídos 25 indivíduos saudáveis, selecionados pelos mesmos critérios de exclusão utilizados para os pacientes com FMJ, 
sem história de dor crônica, sem uso de medicamentos e sem alterações osteomioarticulares.

Os voluntários foram recrutados na comunidade, em famílias de amigos ou indicados por profissionais das instituições onde o estudo foi realizado.

\subsubsection{Critérios de exclusão relacionados à prática de atividade física}

Foram excluídos do estudo os pacientes FMJ e os sujeitos controles saudáveis fisicamente ativos (com prática de exercício físico de moderada ou vigorosa intensidade, pelo menos 60 minutos diários, durante cinco ou mais dias por semana e período inferior a seis meses) ${ }^{30,31}$. Foram aceitos no estudo somente os sujeitos participantes de aulas de educação física na escola, com atividade inferior a 60 minutos e no máximo duas vezes por semana ${ }^{32}$.

\subsubsection{Aspectos éticos}

Este estudo foi aprovado pelos comitês de ética em pesquisa dos quatro serviços universitários (parecer CAPPesq do HC-FMUSP nำ1002/09). O termo de consentimento informado por escrito, apropriado para a idade, foi obtido de todos os participantes e de seus responsáveis legais. 


\subsection{Procedimentos do estudo}

\subsubsection{Dados demográficos}

As medidas antropométricas foram usadas para mostrar pareamento e similaridade que caracterizaram os sujeitos da pesquisa, sendo mensuradas nos grupos de pacientes com FMJ e indivíduos saudáveis.

a. O peso corporal foi medido utilizando uma balança mecânica (Filizola modelo 31-São Paulo, Brasil) com aproximação a 0,1kg, com indivíduos vestidos com camisetas e calções leves.

b. A altura corporal foi medida utilizando o estadiômetro Filizola (modelo 31, São Paulo, Brasil) com aproximação de 0,1 centímetros.

c. O índice de massa corpórea (IMC) foi calculado pela divisão do peso corporal $(\mathrm{Kg})$ pela medida da altura $\left(\mathrm{m}^{2}\right)$. Os valores de referência para obesidade foram IMC $>97$, percentil para idade e sexo (escore $z \geq+2$ e escore $z \leq+3)^{33,34}$.

d. Avaliação específica dos estágios puberais de Tanner classificados em cinco etapas, de acordo com a sequência de eventos puberais e caracteres sexuais secundários resultantes da maturação hormonal ${ }^{35}$. 


\subsubsection{Avaliação inicial da capacidade funcional e qualidade de vida} relacionada à saúde (QVRS), índice de dor e critérios de hipermobilidade articular

a. Capacidade funcional para atividades da vida diária: foi avaliada em pacientes com FMJ, de acordo com a versão brasileira validada do Childhood Health Assessment Questionnaire (CHAQ), composto de oito domínios (vestir, levantar, comer, caminhar, higiene, alcançar, preensão e atividades de vida diária). As pontuações mais baixas indicam melhor capacidade funcional. As pontuações mais altas indicam maior grau de incapacidade física. O escore final consistiu na média das oito áreas (variação de 0 a 3) com zero significando ausência de dificuldade e três incapacidade ${ }^{36}$.

b. Avaliação de QVRS: foi avaliada de acordo com o questionário pediátrico Pediatric Quality of Life InventoryTM Versão 4.0 (Peds $\mathbf{Q L}^{\mathrm{TM}}$ 4.0) foi composto de 23 itens que incluíram avaliações das dimensões física, emocional, social e escolar. Este foi realizado por autoavaliação nas crianças, nos adolescentes e nos responsáveis legais, do grupo de pacientes e controles saudáveis. Foram utilizados os instrumentos validados para o idioma português (Brasil) para as faixas etárias de 8 a 12 anos e de 13 a 18 anos $^{37}$.

c. Intensidade da dor e sintomas: a escala visual analógica (VAS Visual Analog Scale), unidimensional, foi utilizada para mensurar a dor. O nível de dor foi marcado em uma linha horizontal de $10 \mathrm{~cm}$, sem marcações numéricas, indicando 0 = "sem dor" e $10=$ "pior dor 
possível", tendo como base o autorrelato da intensidade da dor na última semana ${ }^{38,39}$.

d. Tender points: Foram mensurados nos 18 pontos dolorosos descritos de acordo com os critérios de FM do ACR, $1990^{40}$.

e. Hipermobilidade articular: a síndrome da hipermobilidade articular benigna foi definida pela avaliação clínica dos pacientes, quando presente aumento da flexibilidade articular em, pelo menos, quatro articulações, diagnosticada de acordo com os critérios de Beighton ${ }^{41}$ ou os critérios de Carter Wilkinson ${ }^{42}$.

\subsubsection{Teste cardiopulmonar de exercício}

O teste cardiopulmonar de exercício ou ergoespirométrico é um método não invasivo capaz de mensurar dinamicamente a resposta fisiológica e metabólica em uma condição de estresse induzida pelo exercício, permitindo a avaliação da função autonômica cardíaca.

Os participantes do estudo, pacientes e controles saudáveis, realizaram o teste cardiopulmonar de exercício em esteira rolante (Centurion, model 200, Micromed, Brasil), utilizando um protocolo de exercício físico máximo progressivo ${ }^{17}$ e cego para os resultados clínicos. As velocidades da esteira $(2,0 ; 2,5 ; 3,0 ; 3,5$; $4,0 ; 4,0 ; 4,0 ; 4,0 ; 5,0 ; 5,4 ; 5,9 ; 6,3 ; 6,3 \mathrm{mph})$ e a inclinação $(0,0 ; 0,0 ; 0,0 ; 0,0$; $0,0 ; 2,5 ; 5,0 ; 7,5 ; 4,0 ; 4,0 ; 4,0 ; 4,0 ; 5,8 \%)$ foram aumentadas progressivamente a cada minuto, até a exaustão. O tempo de duração do teste foi de 8 a 12 minutos, aproximadamente. O período de recuperação foi fixado em 2 minutos. 


\section{a. Avaliação cardiovascular inicial}

A avaliação cardiovascular foi realizada previamente à avaliação ergométrica, com a finalidade de verificar possíveis alterações eletrocardiográficas. Os sujeitos do estudo foram submetidos a eletrocardiograma (ECG) de 12 derivações $\left(D_{1}, D_{2}, D_{3}, A V_{R}, A V_{L}, A V_{F}, V_{1}, V_{2}, V_{3}, V_{4}, V_{5}\right.$ e $\left.V_{6}\right)$, registradas em ECG computadorizado (Ergo PC Elite, InC. Micromed, Brasília, DF, Brazil). A FC foi monitorada continuamente, registrada no repouso, durante o exercício e na fase de recuperação (ECG de 12 derivações).

A pressão arterial (PA) foi medida pelo método auscultatório, imediatamente ao final de cada estágio do teste, utilizando um esfigmomanômetro aneroide (Sphygmomanometer Tycos, EUA). A oximetria de pulso permitiu monitorar a saturação periférica oxigênio em valores $\geq 90 \%$.

\section{b. Avaliação do consumo de oxigênio e determinação dos limiares ventilatórios}

O consumo de oxigênio $\left(\mathrm{VO}_{2}\right.$ STPD), a produção dióxido de carbono $\left(\mathrm{VCO}_{2}\right.$ STPD $)$, assim como a ventilação pulmonar ( $\left.\mathrm{V}_{\mathrm{E} \text { BTPS }}\right)$, e a razão da troca respiratória $\left(\mathrm{RER}=\mathrm{VCO}_{2} / \mathrm{VO}_{2}\right)$ foram avaliados a cada ciclo respiratório, a partir de valores medidos por uma técnica de amostragem "respiração - a respiração" expressa em 30 segundos. Esta avaliação foi realizada por um sistema de calorimetria indireta, sensores de oxigênio $\left(\mathrm{O}_{2}\right)$, dióxido de carbono $\left(\mathrm{CO}_{2}\right)$ e análise computadorizada da troca gasosa (Cortex, modelo Metalyzer III B, Leipzig, Germany. Neste estudo, foi utilizado o pico de VO2 como substituto para $\mathrm{VO}^{2}$ máximo.

$\mathrm{O}$ consumo de oxigênio de pico (pico de $\mathrm{VO}^{2}$ ) foi considerado com base na média dos últimos 30 segundos do teste e foi utilizado para estimar o $\mathrm{VO}^{2}$ 
máximo ${ }^{25,43,44}$. O limiar anaeróbio ventilatório (LAV) foi determinado quando o equivalente ventilatório para $\mathrm{o}_{\mathrm{VO}} \quad \mathrm{VO}_{2}\left(\mathrm{VE} / \mathrm{VO}_{2}\right)$ aumentou sem $\mathrm{o}$ aumento concomitante do equivalente ventilatório para o dióxido de carbono $\left(\mathrm{VE} / \mathrm{VCO}_{2}\right)$.

O ponto de compensação respiratório (PCR) foi determinado quando os equivalentes ventilatórios $\mathrm{VE} / \mathrm{VO}_{2}$ e $\mathrm{VE} / \mathrm{VCO}_{2}$ aumentaram simultaneamente ${ }^{25,45,43,44,46}$. O teste foi considerado completo quando, apesar de encorajados, a criança ou o adolescente não foi capaz de continuar o exercício.

Os critérios utilizados para definir o esforço máximo foram: evidência subjetiva de exaustão e FC pico > 190 batimentos/minuto ou razão de troca respiratória máxima $(\mathrm{RER})>1,00^{47}$. A duração do teste de exercício cardiorrespiratório variou de 8 a 12 minutos.

\section{c. Avaliação da modulação autonômica cardíaca}

A resposta da FC durante o exercício foi avaliada pela reserva cronotrópica $(\mathrm{RC})$ em jovens adolescentes (até 16 anos), como segue:

$$
\begin{aligned}
& \mathrm{RC}=[\mathrm{FC} \text { pico }-\mathrm{FC} \text { repouso } / 208-(0,7 \mathrm{x} \text { idade })-\mathrm{FC} \text { repouso }] \times 100(<16 \text { anos })^{48,49} . \\
& \mathrm{RC}=[\mathrm{FC} \text { pico }-\mathrm{FC} \text { repouso } /(220-\text { idade })-F C \text { repouso }] \times 100(>16 \text { anos })^{50} .
\end{aligned}
$$

A recuperação $\mathrm{FC}$ foi definida como a diferença entre $\mathrm{FC}$ pico e $\mathrm{FC}$ no primeiro minuto $(\triangle F C R 1)$ e no segundo minuto $(\triangle F C R 2)$ após exercício. A mudança absoluta $(\Delta)$ foi usada para calcular a diferença entre FC pico de exercício, no primeiro e no segundo minutos após o teste de exercício. A mudança relativa no $\mathrm{FC}(\Delta \%)$ foi calculada para os intervalos entre repouso ao $L A$, repouso ao PCR, e repouso ao pico do exercício. 


\subsection{Análise estatística}

Todas as análises estatísticas foram realizadas com o software R 3.1.1.

Os dados foram apresentados em mediana (variação), ou média \pm SD para variáveis contínuas, de acordo com a distribuição anormal ou normal, respectivamente. Os dados foram apresentados em número (porcentagens), para variáveis categóricas, e comparados pelo teste de Fisher. Para variáveis contínuas, os dados foram comparados por meio de testes t de Mann-Whitney ou, respectivamente, para avaliar diferenças entre os pacientes FMJ e os controles saudáveis. Em pacientes com FMJ, o coeficiente de correlação de Pearson foi utilizado para identificar as variáveis correlacionadas à graduação do teste cardiorrespiratório de exercício ao escore de dor, do QVRS e dos parâmetros CHAQ. Os valores de $p$ inferiores a 0,05 foram considerados significativos. 
4 RESULTADOS 
Dados demográficos e estágio puberal de Tanner, avaliados dos pacientes com FMJ e controles saudáveis, são apresentados na Tabela 1. A média da idade atual foi semelhante nos pacientes com FMJ e controles saudáveis [15 (11-17) vs. $15(11-17)$ anos, $\mathrm{p}=0,890)]$, assim como peso corporal $(p=0,159)$, altura $(p=0,264)$ e IMC $(p=0,332)$. As frequências de sexo feminino $(72 \%$ vs. $72 \%, p=1,000)$ e estágio puberal de Tanner $(p=0,822)$ foram semelhantes em ambos os grupos (Tabela 1).

Tabela 1 - Dados demográficos e estágio puberal de Tanner em pacientes com fibromialgia juvenil primária (FMJP) e controles saudáveis.

\begin{tabular}{lccc}
\hline Variáveis & $\begin{array}{c}\text { Pacientes } \\
\text { com FMJ } \\
(\mathbf{n = 2 5})\end{array}$ & $\begin{array}{c}\text { Controles } \\
\text { saudáveis } \\
(\mathbf{n}=25)\end{array}$ & $\boldsymbol{p}$ \\
\hline Dados demográficos & $15(11-17)$ & $15(11-17)$ & 0,890 \\
Idade atual, anos & $54,5(37,7-79,8)$ & $55,7(30,3-74,7)$ & 0,159 \\
Peso corporal, kg & $159,0(142,5-170,0)$ & $163,0(141,0-178,5)$ & 0,264 \\
Altura, m & $20,4(15,7-27,7)$ & $21,0(15,7-27,7)$ & 0,332 \\
Índice de massa corporal, kg/m² & $18(72)$ & $18(72)$ & 1,000 \\
Gênero feminino & & & 0,822 \\
Estágio puberal de Tanner & $0(0)$ & $1(4)$ & \\
I & $7(28)$ & $6(24)$ & \\
II & $5(20)$ & $3(12)$ & \\
III & $6(24)$ & $9(36)$ & \\
IV & $7(28)$ & $6(24)$ & \\
V & & & \\
\hline
\end{tabular}

Os resultados foram apresentados em mediana (valores mínimo - máximo) e n (\%). 
Os dados descritos da avaliação clínica dos pacientes com FMJ são apresentados na Tabela 2.

Tabela 2 - Estatística descritiva da avaliação clinica e funcional dos pacientes com fibromialgia juvenil primária (FMJP).

Variáveis

Pacientes $(n=25)$

\section{Avaliação da dor e sintomas clínicos}

VAS - Intensidade da dor (0 -10)

$6,2(4,5-9,1)$

VAS - Sintomas *

$5,9(2,5-8,3)$

Tender points (0-18), (ACR, 1990)

$15(11-18)$

Critérios de Wolfe, 2010, escore

Índice de dor difusa, (0 - 19)

$13(10-16)$

Sintomas principais, $(0-12)$

$8(3-9)$

Sintomas somáticos, $(0$ - 3)

$3(3-3)$

Índice de severidade dos sintomas

$10(6-12)$

Sintomas somáticos, ( ํo de sintomas/paciente)

$16(10-25)$

Frequência da dor, no, (\%)

1 - 3 vezes por semana

4 - 6 vezes por semana

Todos os dias

Drogas analgésicas, no, (\%)

Analgésico (dipirona e paracetamol)

Anti-inflamatório

Opióide

CHAQ - escore (0 - 3)

Síndrome de hipermobilidade articular benigna, $\mathrm{n}^{\circ}$, (\%)

Tempo do início dos sinais de FMJ (meses) $24(6-72)$

Os resultados foram apresentados em mediana (valores mínimo - máximo), número e (\%).

CHAQ - Childhood Health Assessment Questionnaire, VAS - Visual Analog Scale.

*fadiga, problemas com sono, ansiedade, nervosismo, dor abdominal, cefaleia. 
A mediana dos parâmetros QVRS (escore total, saúde física e saúde psicossocial) foi significativamente menor nos pacientes FMJ, quando comparados aos controles saudáveis (Tabela 3).

Tabela 3 - Dados qualidade de vida relacionada à saúde (QVRS) em pacientes com fibromialgia juvenil primária (FMJP) e controles saudáveis

\begin{tabular}{lccc}
\hline Variáveis & $\begin{array}{c}\text { Pacientes } \\
\text { com FMJ } \\
(\mathbf{n = 2 5})\end{array}$ & $\begin{array}{c}\text { Controles } \\
\text { saudáveis } \\
(\mathbf{n = 2 5})\end{array}$ & $\boldsymbol{p}$ \\
\hline QVRS (0-100) & & & \\
Adolescentes & $61,88(32,34-72,94)$ & $84,38(67,97-89,69)$ & $<0,001$ \\
Escore total & $61,88(32,34-72,97)$ & $87,50(68,75-93,75)$ & $<0,001$ \\
Saúde física & $61,67(30,00-73,33)$ & $83,33(66,67-88,33)$ & $<0,001$ \\
Saúde psicossocial & & & \\
Pais & $58,59(32,97-74,69)$ & $82,66(74,06-90,94)$ & $<0,001$ \\
Escore total & $53,13(15,63-84,38)$ & $87,50(78,13-100)$ & $<0,001$ \\
Saúde física & $60,00(33,33-76,67)$ & $80,00(66,67-90)$ & $<0,001$ \\
Saúde psicossocial & & & \\
\hline
\end{tabular}

Os resultados foram apresentados em mediana (valores mínimo - máximo) e n (\%).

QVRS - qualidade de vida relacionada à saúde.

A mediana da FC pico [181 (150-198) vs. 197 (181-202) bpm, $p<0,001]$, FC pico [92 (75-99) vs. 99 (91-102)\%, p <0,001], reserva cronotrópica [84 (53-98) vs. 99 (84-103)\%, p<0,001], FC repouso ao PCR [83 (45-161) vs. 96 (46-160) bpm, $p=0,008]$ e FC repouso ao pico [96 (65-181) vs. 127 (61-185) bpm, $p=0,010]$ foi significativamente menor nos pacientes com FMJ em comparação com controles saudáveis. A mediana do $\triangle \mathrm{FCR} 1$ [15 (3-39) vs. 35 (9-52) bpm, $p<0,001], \triangle F C R 2$ [37 (20-57) vs. $51(32-94)$ bpm, p<0,001], pico VO2 [32,34 $(24,24-39,65)$ vs. $36,4(28,56-52,71) \mathrm{ml} / \mathrm{kg} / \mathrm{min}, \mathrm{p}=0,005]$; velocidade de pico 
[5 (4-6,3) vs. $5,9(4,0-6,3) \mathrm{mph}, \mathrm{p}=0,001]$, tempo de exaustão $[11,5(8,5-14,5)$ vs. $14(11-18)$ minutos, $p<0,001]$ e a capacidade de trabalho $[3,37(2,04-5,6)$ vs. 3,89 $(2,91-6,55) \mathrm{W} / \mathrm{kg}, \mathrm{p}=0,006]$ também foram significativamente menores nos pacientes FMJ em comparação com controles saudáveis. A frequência da incompetência cronotrópica foi significativamente maior em pacientes FMJ versus controles saudáveis $(\mathrm{p}=0,0006)$ (Tabela 4$)$.

Tabela 4 - Dados cardiovasculares mensurados durante e após exercício de esforço máximo em pacientes com Fibromialgia juvenil primária (FMJP) e controles saudáveis.

\begin{tabular}{|c|c|c|c|}
\hline Variáveis & $\begin{array}{c}\text { Pacientes } \\
\text { FMJ } \\
(n=25)\end{array}$ & $\begin{array}{c}\text { Controles } \\
\text { Saudáveis } \\
(n=25)\end{array}$ & $p$ \\
\hline \multicolumn{4}{|l|}{ Cardiovasculares } \\
\hline FC repouso, bpm & $90(92-120)$ & $85(67-124)$ & 0,286 \\
\hline FC pico predita, bpm & $198(196-201)$ & $199(196-201)$ & 0,742 \\
\hline FC pico, bpm & $181(150-198)$ & $197(181-202)$ & $<0,001$ \\
\hline FC pico, \% & $92(75-99)$ & $99(91-102)$ & $<0,001$ \\
\hline Reserva Cronotrópica, \% & $84(53-98)$ & $99(84-103)$ & $<0,001$ \\
\hline Incompetência cronotrópica, $\leq 80 \%$ & $10(40 \%)$ & $0(0)$ & 0,0006 \\
\hline \multicolumn{4}{|l|}{$\mathrm{FC}, \%$} \\
\hline Repouso - LA & $41(17-66)$ & $40(18-121)$ & 1,000 \\
\hline Repouso - PCR & $83(45-161)$ & $96(46-160)$ & 0,008 \\
\hline Repouso - Pico & $96(65-181)$ & $127(61-185)$ & 0,010 \\
\hline \multicolumn{4}{|l|}{ FC repouso, bpm } \\
\hline$\Delta \mathrm{FCR} 1$ & $15(3-39)$ & $35(9-52)$ & $<0,001$ \\
\hline$\Delta \mathrm{FCR} 2$ & $37(20-57)$ & $51(32-94)$ & $<0,001$ \\
\hline \multicolumn{4}{|l|}{ Respiratórias } \\
\hline $\mathrm{VO}_{2}$ de pico, $\mathrm{ml} / \mathrm{kg} / \mathrm{min}$ & $32,34(24,24-39,65)$ & $36,4(28,56-52,71)$ & 0,005 \\
\hline $\operatorname{RTR}\left(\mathrm{CO}_{2} / \mathrm{O}_{2}\right)$ pico & $1,04(0,95-1,31)$ & $1,10(0,93-1,24)$ & 0,050 \\
\hline \multicolumn{4}{|l|}{ Desempenho máximo } \\
\hline Velocidade de pico, mph & $5(4,0-6,3)$ & $5,9(4,0-6,3)$ & 0,001 \\
\hline Inclinação máxima, \% & $4(2,5-12)$ & $4(4-9,4)$ & 0,399 \\
\hline Tempo de exaustão, minutos & $11,5(8,5-14,5)$ & $14(11-18)$ & $<0,001$ \\
\hline Capacidade de trabalho, W/kg & $3,37(2,04-5,6)$ & $3,89(2,91-6,55)$ & 0,006 \\
\hline
\end{tabular}

Os resultados foram apresentados em mediana (valores mínimo - máximo) e n (\%). 
Foram observadas correlações positivas entre os parâmetros QVRS (de acordo com o autorrelato dos pacientes FMJ e dos pais) e: velocidade de pico, tempo de exaustão, FC pico, reserva cronotrópica, $\mathrm{VO}^{2}$ de pico, capacidade de trabalho, $\triangle \mathrm{FCR} 1$ e $\triangle \mathrm{FCR} 2$ ( $\mathrm{r}$ variou de 0,291 a + 0,619) (Tabela 5).

Correlações negativas foram evidenciadas entre os escores do CHAQ e: tempo de exaustão, FC pico, reserva cronotrópica, $\mathrm{VO}^{2}$ de pico e capacidade de trabalho ( $r$ variou de $-0,402$ para $-0,613$ ) em pacientes FMJ. Correlação negativa foi observada entre o escore de dor e a inclinação máxima, $r=-0,443$ (Tabela 5). 
Tabela 5 - Coeficiente de correlação de Pearson's para os parâmetros de QVRS, CHAQ, escore de dor e dados cardiorrespiratórios mensurados nos pacientes com fibromialgia juvenil primária

\begin{tabular}{|c|c|c|c|c|c|c|c|c|c|}
\hline Variáveis & $\begin{array}{c}\text { Velocidade } \\
\text { pico, } \\
\text { mph }\end{array}$ & $\begin{array}{c}\text { Tempo } \\
\text { exaustão } \\
\text { minutos }\end{array}$ & $\begin{array}{c}\text { FC } \\
\text { de pico } \\
\text { bpm }\end{array}$ & $\begin{array}{c}\text { FC } \\
\text { de pico } \\
\%\end{array}$ & $\begin{array}{c}\text { Reserva } \\
\text { cronotrópica } \\
\%\end{array}$ & $\begin{array}{c}\mathrm{VO}_{2} \\
\text { de pico } \\
\mathrm{ml} / \mathrm{kg} \\
/ \mathrm{min}\end{array}$ & $\begin{array}{c}\text { Capacidade } \\
\text { de trabalho } \\
\text { W/kg }\end{array}$ & $\triangle \mathrm{FCR} 1$ & $\triangle \mathrm{FCR} 2$ \\
\hline \multicolumn{10}{|l|}{ QVRS } \\
\hline \multicolumn{10}{|l|}{ Pacientes } \\
\hline Saúde física & $+0,378^{*}$ & $+0,520^{*}$ & $+0,493^{*}$ & $+0,490^{*}$ & $+0,505^{*}$ & $+0,384^{*}$ & $+0,298^{*}$ & $+0,602^{*}$ & $+0,486^{*}$ \\
\hline Saúde psicossocial & $+0,291^{*}$ & $+0,471^{*}$ & $+0,427^{*}$ & $+0,434^{*}$ & $+0,444^{*}$ & $+0,310^{*}$ & - & $+0,565^{\star}$ & $+0,416^{*}$ \\
\hline Escore total & $+0,359^{*}$ & $+0,513^{*}$ & $+0,470^{*}$ & $+0,468^{*}$ & $+0,483^{*}$ & $+0,376^{*}$ & $+0,311^{*}$ & $+0,590^{*}$ & $+0,459^{*}$ \\
\hline \multicolumn{10}{|l|}{ Pais } \\
\hline Saúde física & $+0,308^{*}$ & $+0,452^{*}$ & $+0,429^{*}$ & $+0,444^{*}$ & $+0,450^{*}$ & $+0,330^{*}$ & - & $+0,619^{*}$ & $+0,467^{*}$ \\
\hline Saúde psicossocial & $+0,345^{\star}$ & $+0,486^{*}$ & $+0,483^{*}$ & $+0,512^{*}$ & $+0,521^{*}$ & - & - & $+0,461^{*}$ & $+0,313^{*}$ \\
\hline Escore total & $+0,349^{*}$ & $+0,503^{*}$ & $+0,490^{*}$ & $+0,516^{*}$ & $+0,525^{\star}$ & - & - & $+0,536^{*}$ & $+0,378^{*}$ \\
\hline Escore CHAQ & - & $-0,422^{*}$ & $-0,521^{\star}$ & $-0,446^{*}$ & $-0,525^{\star}$ & $-0,613^{*}$ & $-0,402^{*}$ & - & - \\
\hline
\end{tabular}

Nível de significância $\left(^{*}\right) p<5 \%$.

FC - frequência cardíaca; pico - pico do exercício; $\triangle F C R 1$ - delta da frequência cardíaca de recuperação no primeiro minuto; $\Delta$ HRR2 - delta da frequência cardíaca de recuperação no segundo minuto; $\mathrm{VO}_{2}$ - consumo de oxigênio; bpm - batimentos por minuto; $\mathrm{QVRS}$ - qualidade de vida relacionada à saúde; CHAQ - Childhood Health Assessment Questionnaire. 
Este estudo foi o primeiro que avaliou teste de exercício progressivo em pacientes FMJ no momento do diagnóstico e mostrou evidente incompetência cronotrópica e atenuada recuperação da FC, indicando uma disfunção autonômica. Também foram observadas reduzidas a capacidade cardiorrespiratória e a tolerância ao exercício.

Os pontos fortes deste estudo multicêntrico foram a inclusão dos critérios diagnósticos para FM do American College of Rheumatology ${ }^{29}$, também recentemente estudados na população $F M J^{51}$, bem como a utilização de parâmetros padronizados para execução do teste de exercício cardiopulmonar progressivo ${ }^{51,53,55,73}$. A inclusão apenas de pacientes com FMJ e controles saudáveis com variáveis similares (idade atual, sexo, estágio puberal de Tanner e IMC) e ainda que praticaram exclusivamente aulas de educação física na escola foi essencial, pois, de fato, essas variáveis podem influenciar a capacidade cardiorrespiratória. O valor da FC de repouso garantiu a similaridade do condicionamento físico entre pacientes e controles saudáveis. No presente estudo, foram excluídos pacientes com FMJ que faziam uso de medicamentos, tais como antidepressivos e anti-hipertensivos, cuja ação pudesse induzir a resposta cronotrópica ao exercício.

A FMJ tem prevalência relatada entre $0 \%$ e $6,1 \%$ em diferentes populações, incluindo estudos realizados no Brasil ${ }^{1,2,3,51}$. Esta doença crônica é caracterizada por dor músculo-esquelética generalizada, incapacidade funcional ${ }^{7}$ e evidente impacto na QVRS dos pacientes e de seus cuidadores ${ }^{10,11}$, tal como 
foi observado neste estudo. As alterações mecânicas na articulação podem afetar a marcha, a postura, a força e a atividade física ${ }^{1,53}$, assim, e a abordagem multidisciplinar é necessária para o tratamento de pacientes com $\mathrm{FM}^{9}$.

Os tratamentos mais utilizados para os pacientes JFM são administração de antidepressivos e de drogas anticonvulsivantes ${ }^{54}$ e terapia cognitivocomportamental $^{55,56,57}$. Estudos têm evidenciado que exercício aeróbico é uma terapia eficaz e segura para pacientes $F M^{58.59 .60}$. No entanto, é importante destacar que estas terapias não foram administradas em nossos pacientes com FMJ, uma vez que estes foram avaliados no momento do diagnóstico, como foi descrito.

Outro aspecto relevante, evidenciado neste estudo, foi que os pacientes com FMJ tinham menor capacidade funcional e níveis inferiores de atividade física, possivelmente devido à reduzida capacidade cardiorrespiratória, como também foi observado em pacientes com lúpus eritematoso sistêmico juvenil ${ }^{61}$, síndrome do anticorpo antifosfolípide ${ }^{25}$ e dermatomiosite juvenil ${ }^{62,26}$. Além disso, o presente estudo evidenciou que pacientes com FMJ tiveram resposta anormal da FC durante e após o teste de exercício cardiopulmonar (exemplificada pela reserva cronotrópica reduzida e pela recuperação atenuada da FC). Este resultado foi também observado em uma população de mulheres adultas com $\mathrm{FM}^{12}$.

Um aspecto interessante é que a reserva cronotrópica e a recuperação da FC são marcadores de risco cardiovascular não-invasivos e indicam disautonomia, sugerindo comprometimento da resposta autonômica cardíaca ${ }^{25,61}$. Além disto, a redução da aptidão cardiorrespiratória e a disfunção do sistema nervoso autônomo têm sido associadas com maior risco cardiovascular na 
população adulta ${ }^{63,64}$. A incompetência cronotrópica foi observada em mais de um terço dos pacientes FMJ no presente estudo. Esse problema foi relatado em pacientes adultos com FM, quando a IC foi observada em metade da amostra de pacientes do estudo ${ }^{12}$. Este importante parâmetro tem sido associado com maior mortalidade cardiovascular em indivíduos saudáveis ${ }^{43}$. Estudos prospectivos serão necessários para determinar o poder preditivo da resposta cronotrópica como um fator de risco cardiovascular em adolescentes com FMJ.

O presente estudo estendeu uma pesquisa anterior, que foi realizada em pacientes adultos com FM submetidos ao teste cardiorrespiratório ${ }^{12}$. No presente estudo, foram evidenciadas correlações fracas e moderadas da reserva cronotrópica e da capacidade aeróbia com parâmetros da QVRS. No entanto, as correlações foram inversamente proporcionais à incapacidade funcional e ao índice de dor. Assim como, também, foi inversamente proporcional à inclinação máxima no pico do exercício, sugerindo que nossos pacientes com FMJ tiveram maior grau de dificuldade para realizar as atividades físicas e foram incapazes de atingir o teste máximo cardiorrespiratório.

Neste estudo, não foi observada alta frequência de síndrome de hipermobilidade articular benigna concomitante com FMJ. Este aspecto foi diferente de um estudo anterior, no qual aproximadamente metade dos pacientes adolescentes apresentou as duas doenças simultaneamente ${ }^{65}$. As manifestações extra-articulares da síndrome de hipermobilidade articular benigna podem incluir também anormalidades do sistema nervoso autônomo ${ }^{66}$. No entanto, apenas um de nossos pacientes com FMJ tinha disautonomia e síndrome da hipermobilidade articular benigna. 
Os critérios restritivos de inclusão e exclusão desse estudo limitaram o número de pacientes. No entanto, esses mesmos critérios garantiram a inclusão de pacientes com o diagnóstico inicial de FMJ primária, sem outras doenças crônicas pediátricas ou em uso de tratamento prévio (tais como: medicamentos antidepressivos ou terapia cognitivo-comportamental) que pudessem interferir no resultado do estudo.

Este estudo apresentou algumas limitações. A composição corporal (massa gorda, massa magra e gordura percentual) não foi mensurada, sendo que de fato, a adiposidade corporal pode contribuir para modulação autonômica cardíaca em crianças e adolescentes obesos ${ }^{67,68}$. No entanto, o IMC foi similar em pacientes com FMJ e controles saudáveis. Não foram identificados obesos (valores de referência para obesidade da OMS, 2007) ${ }^{64,65}$, e essas variáveis não interferiram nos resultados da resposta autonômica cardíaca de ambos os grupos. Além disso, distúrbios do sono não foram avaliados nos dois grupos.

Os parâmetros cardiopulmonares e de condicionamento aeróbico geral melhoraram após o programa de treinamento aeróbio supervisionado em algumas doenças crônicas pediátricas ${ }^{56,57,84,85}$. Neste estudo, o teste de exercício cardiorrespiratório não foi avaliado prospectivamente nos pacientes com FMJ após um programa de treinamento de atividade física.

O exercício aeróbio de moderada intensidade é indubitavelmente indicado para todos os pacientes com $\mathrm{FMJ}^{83}$, pois pode modular a capacidade autonômica cardíaca e reduzir o risco cardiovascular, conforme demonstrou um estudo em pacientes com lúpus eritematoso sistêmico juvenil ${ }^{83}$. No presente estudo, foram identificadas a incompetência cronotrópica (marcador de risco 
cardiovascular independente de outros fatores de risco) e a intolerância ao exercício. Entretanto, modulação autonômica ao exercício físico e eventos cardiovasculares ainda não são conhecidos em pacientes com FMJ e necessitarão de futuros estudos longitudinais. 
1. Incompetência cronotrópica e atenuada recuperação da frequência cardíaca, indicando disfunção autonômica, foram observadas em pacientes FMJ no momento do diagnóstico.

2. Correlações entre os parâmetros do teste cardiopulmonar de exercício com a dor, a capacidade funcional e a qualidade de vida relacionada à saúde foram encontradas em pacientes com FMJ. 
1. Kashikar-Zuck S, Ting TV. Juvenile fibromyalgia: current status of research and future developments. Nature Reviews Rheumatology. 2014;10(2):89-96.

2. Zapata AL, Moraes AJP, Leone C, Doria U, Silva CAA. Pain and musculoskeletal pain syndromes in adolescents. Journal of Adolescent Health. 2006;38(6):769-71.

3. Zapata AL, Moraes AJP, Leone C, Doria U, Silva CAA. Pain and musculoskeletal pain syndromes related to computer and video game use in adolescents. European Journal of Pediatrics. 2006;165(6):408-14.

4. Kashikar-Zuck S, Parkins IS, Graham TB, Lynch AM, Passo M, Johnston M, et al. Anxiety, mood, and behavioral disorders among pediatric patients with juvenile fibromyalgia syndrome. Clin J Pain. 2008;24(7):620-6.

5. Choy EH, Mease PJ. Key Symptom Domains to Be Assessed in Fibromyalgia (Outcome Measures in Rheumatoid Arthritis Clinical Trials). Rheumatic Disease Clinics of North America. 2009;35(2):329-+.

6. Michels H, Gerhold K, Hafner R, Hauser W, Illhardt A, Monkemoller K, et al. Juvenile fibromyalgia syndrome. Schmerz. 2008;22(3):339-48.

7. Buskila D, Press J, Gedalia A, Klein M, Neumann L, Boehm R, et al. Assessment of nonarticular tenderness and prevalence of fibromyalgia in children. Journal of Rheumatology. 1993;20(2):368-70.

8. Clark P, Burgos-Vargas R, Medina-Palma C, Lavielle P, Marina FF. Prevalence of fibromyalgia in children: A clinical study of Mexican children. Journal of Rheumatology. 1998;25(10):2009-14. 
9. Tesher MS. Juvenile Fibromyalgia: A Multidisciplinary Approach to Treatment. Pediatr Ann. 2015;44(6):e136-41.

10. Kashikar-Zuck S, Zafar M, Barnett KA, Aylward BS, Strotman D, Slater SK, et al. Quality of life and emotional functioning in youth with chronic migraine and juvenile fibromyalgia. Clin J Pain. 2013;29(12):1066-72.

11. Kashikar-Zuck S, Cunningham N, Sil S, Bromberg MH, Lynch-Jordan AM, Strotman D, et al. Long-Term Outcomes of Adolescents With Juvenile-Onset Fibromyalgia in Early Adulthood. Pediatrics. 2014;133(3):E592-E600.

12. da Cunha Ribeiro RP, Roschel H, Artioli GG, Dassouki T, Perandini LA, Calich $A L$, et al. Cardiac autonomic impairment and chronotropic incompetence in fibromyalgia. Arthritis Res Ther. 2011;13(6):R190.

13. Bardal EM, Olsen TV, Ettema G, Mork PJ. Metabolic rate, cardiac response, and aerobic capacity in fibromyalgia: a case-control study. Scandinavian Journal of Rheumatology. 2013;42(5):417-20.

14. Kulshreshtha P, Deepak KK. Autonomic nervous system profile in fibromyalgia patients and its modulation by exercise: a mini review. Clinical Physiology and Functional Imaging. 2013;33(2):83-91.

15. Martinez-Lavin M, Hermosillo AG. Autonomic nervous system dysfunction may explain the multisystem features of fibromyalgia. Seminars in Arthritis and Rheumatism. 2000;29(4):197-9.

16. Friederich HC, Schellberg D, Mueller K, Bieber C, Zipfel S, Eich W. Stress and autonomic dysregulation in patients with fibromyalgia syndrome. Schmerz. 2005;19(3):185-+.

17. Cohen H, Neumann L, Kotler M, Buskila D. Autonomic nervous system derangement in fibromyalgia syndrome and related disorders. Isr Med Assoc J. $2001 ; 3(10): 755-60$. 
18. Furlan R, Colombo S, Perego F, Atzeni F, Diana A, Barbic F, et al. Abnormalities of cardiovascular neural control and reduced orthostatic tolerance in patients with primary fibromyalgia. Journal of Rheumatology. 2005;32(9):1787-93.

19.Lauer MS. Heart Rate Response in Stress Testing: Clinical Implications. https://www.uthsc.edu/cardiology/articles/HRresponse\%20in\%20ETT.pdf (accessed 28.04.2016).

20. Ellestad MH, Wan MKC. Predictive implications of stress testing - follow-up of 2700 subjects after maximum treadmill stress testing. Circulation. 1975;51(2):363-9.

21.Lauer MS, Francis GS, Okin PM, Pashkow FJ, Snader CE, Marwick TH. Impaired chronotropic response to exercise stress testing as a predictor of mortality. Jama-Journal of the American Medical Association. 1999;281(6):524-9.

22. Cole CR, Foody JM, Blackstone EH, Lauer MS. Heart rate recovery after submaximal exercise testing as a predictor of mortality in a cardiovascularly healthy cohort. Annals of Internal Medicine. 2000;132(7):552-5.

23. Singh TP, Rhodes J, Gauvreau K. Determinants of heart rate recovery following exercise in children. Medicine and Science in Sports and Exercise. 2008;40(4):601-5.

24.Baba R, Iwagaki S, Tauchi N, Tsurusawa M. Is the chronotropic index applicable to children and adolescents? Circulation Journal. 2005;69(4):471-4. 56.

25. do Prado DL, Gualano B, Miossi R, Sá-Pinto A, Lima F, Roschel H, Borba E, Bonfá E. Abnormal chronotropic reserve and heart rate recovery in patients with SLE: a case-control study. Lupus. 2011 Jun;20(7):717-20. 
26. Omori CH, Almeida Silva CA, Elias Sallum AM, Rodrigues Pereira RM, de Sa Pinto $A L$, Roschel $\mathrm{H}$, et al. Exercise Training in Juvenile Dermatomyositis. Arthritis Care \& Research. 2012;64(8):1186-94. doi: 10.1002/acr.21684.

27. Liem RI, Reddy M, Pelligra SA, Savant AP, Fernhall B, Rodeghier M. Reduced fitness and abnormal cardiopulmonary responses to maximal exercise testing in children and young adults with sickle cell anemia (vol 3, e12338, 2015). Physiological Reports. 2016;4(1):1. doi: 10.14814/phy2.12680.

28.van de Weert-van Leeuwen PB, Slieker MG, Hulzebos HJ, Kruitwagen C, van der Ent CK, Arets HGM. Chronic infection and inflammation affect exercise capacity in cystic fibrosis. European Respiratory Journal. 2012;39(4):893-8.

29. Wolfe F, Clauw DJ, Fitzcharles MA, Goldenberg DL, Katz RS, Mease P, et al. The American College of Rheumatology Preliminary Diagnostic Criteria for Fibromyalgia and Measurement of Symptom Severity. Arthritis Care \& Research. 2010;62(5):600-10.

30. World Health Organization Global recommendations on physical activity for health. Geneve: WHO;2010. http://www.ncbi.nlm.nih.gov/books/NBK305060/ (Accessed 08 Apr 2016).

31. Cavil N, Maibach EW. VERB (TM) - Demonstrating a viable national option for promoting physical activity among our children. American Journal of Preventive Medicine. 2008;34(6):S173-S4.

32. Center for Disease Control and Prevention. [Accessed 08 Apr 2016] Division of Nutrition, Physical Activity, and Obesity. How much physical activity do children need? http://www.cdc.gov/physicalactivity/everyone/guidelines/children.html. 
33. Onis M, Onyango A, Borghi E, Siyam A, Pinol A, Garza C, et al. Enrolment and baseline characteristics in the WHO Multicentre Growth Reference Study. Acta Paediatrica. 2006;95:7-15.

34. World Health Organization, WHO multicentre growth reference study, Child growth standards.http://www.who.int/childgrowth/standards/bmi_for_age/en/, [Accessed 08 Apr 2016.

35. Tanner J M: Growth at adolescence. 2 edition. Edited by: Oxford: Blackwell Scientific Publications; 1962: p. 28-39.

36. Machado CS, Ruperto N, Silva CH, Ferriani VP, Roscoe I, Campos LM, et al. The Brazilian version of the Childhood Health Assessment Questionnaire (CHAQ) and the Child Health Questionnaire (CHQ). Clin Exp Rheumatol. 2001;19(4 Suppl 23):S25-9.

37. Klatchoian DA, Len CA, Terreri MT, Silva M, Itamoto C, Ciconelli RM, et al. Quality of life of children and adolescents from São Paulo: reliability and validity of the Brazilian version of the Pediatric Quality of Life Inventory version 4.0 Generic Core Scales. J Pediatr (Rio J). 2008;84(4):308-15.

38. McGrath PJ, Walco GA, Turk DC, Dworkin RH, Brown MT, Davidson K, et al. Core outcome domains and measures for pediatric acute and chronic/recurrent pain clinical trials: PedIMMPACT recommendations. Journal of Pain. 2008;9(9):771-83.

39.Gift AG. VISUAL ANALOG SCALES - measurement of subjective phenomena. Nursing Research. 1989;38(5):286-8.

40. Wolfe F, Smythe HA, Yunus MB, Bennett RM, Bombardier C, Goldenberg $\mathrm{DL}$, et al. The american-college-of-rheumatology 1990 criteria for the classification of fibromyalgia - report of the multicenter criteria committee. Arthritis and Rheumatism. 1990;33(2):160-72. 
41. Beighton $P$, Solomon L, Soskolne CL. ARTICULAR MOBILITY IN AN AFRICAN POPULATION. Annals of the Rheumatic Diseases. 1973;32(5):413-8.

42. Carter C, Wilkinson J. Persistent joint laxity and congenital dislocation of the hip. Journal of Bone and Joint Surgery-British Volume. 1964;46(1):40-5.

43.Braga A, Nunes N: Avaliação cardiopulmonar. In Cardiologia do Exercício: do Atleta ao Cardiopata. 1 edition. Edited by: Negrão CE, Baretto ACP. São Paulo, Brazil: Manole; 2005:128-147.

44. Miossi R, Benatti FB, Pinto ALD, Lima FR, Borba EF, Prado DML, et al. Using Exercise Training to Counterbalance Chronotropic Incompetence and Delayed Heart Rate Recovery in Systemic Lupus Erythematosus: A Randomized Trial. Arthritis Care \& Research. 2012;64(8):1159-66.

45. Wasserman K, Hansen JE, Suedy, Whipp BJ - eds: Principles of Exercise Testing and Interpretation. Philadelphia: Lca and Febiger, 1987; 47-57.

46. Hebestreit H, Staschen B, Hebestreit A. Ventilatory threshold: a useful method to determine aerobic fitness in children? Medicine and Science in Sports and Exercise. 2000;32(11):1964-9.

47. Rowland TW. Aerobic exercise testing protocols. Pediatric Laboratory Exercise Testing: Clinical Guidelines. 1993:19-41.

48. Machado FA, Denadai BS. Validity of maximum heart rate prediction equations for children and adolescents. Arq Bras Cardiol. 2011;97(2):136-40.

49. Tanaka H, Monahan KD, Seals DR. Age-predicted maximal heart rate revisited. J Am Coll Cardiol. $2001 ; 37(1): 153-6$.

50.Brubaker PH, Kitzman DW. Chronotropic incompetence: causes, consequences, and management. Circulation. 2011;123(9):1010-20. 
51.Ting TV, Barnett K, Lynch-Jordan A, Whitacre C, Henrickson M, KashikarZuck S. 2010 American College of Rheumatology Adult Fibromyalgia Criteria for Use in an Adolescent Female Population with Juvenile Fibromyalgia. Journal of Pediatrics. 2016;169:181-+.

52. Neder L, Rondon DA, Cury SS, da Silva CA. Musculoskeletal manifestations and autoantibodies in children and adolescents with leprosy. Jornal de Pediatria. 2014;90(5):457-63.

53.Sil S, Thomas S, DiCesare C, Strotman D, Ting TV, Myer G, et al. Preliminary evidence of altered biomechanics in adolescents with juvenile fibromyalgia. Arthritis Care Res (Hoboken). 2015;67(1):102-11.

54. Verkamp EK, Flowers SR, Lynch-Jordan AM, Taylor J, Ting TV, KashikarZuck S. A Survey of Conventional and Complementary Therapies Used by Youth with Juvenile-Onset Fibromyalgia. Pain Management Nursing. 2013;14(4):E244-E50.

55. Sil S, Arnold LM, Lynch-Jordan A, Ting TV, Peugh J, Cunningham N, et al. Identifying treatment responders and predictors of improvement after cognitive-behavioral therapy for juvenile fibromyalgia. Pain. 2014;155(7):1206-12.

56. Sherry DD. Physical activity levels in the treatment of juvenile fibromyalgia. Nat Rev Rheumatol. 2013;9(1):8-9. doi: 10.1038/nrrheum.2012.193.

57. Kashikar-Zuck S, Ting TV, Arnold LM, Bean J, Powers SW, Graham TB, et al. Cognitive behavioral therapy for the treatment of juvenile fibromyalgia: a multisite, single-blind, randomized, controlled clinical trial. Arthritis Rheum. 2012;64(1):297-305.

58. Gualano B, Pinto AL, Perondi MB, Roschel H, Sallum AM, Hayashi AP, et al. Therapeutic effects of exercise training in patients with pediatric rheumatic diseases. Rev Bras Reumatol. 2011;51(5):490-6. 
59. Gualano B, Sá Pinto AL, Perondi B, Leite Prado DM, Omori C, Almeida RT, et al. Evidence for prescribing exercise as treatment in pediatric rheumatic diseases. Autoimmun Rev. 2010;9(8):569-73.

60.Stephens S, Feldman BM, Bradley N, Schneiderman J, Wright V, SinghGrewal D, et al. Feasibility and effectiveness of an aerobic exercise program in children with fibromyalgia: results of a randomized controlled pilot trial. Arthritis Rheum. 2008;59(10):1399-406.

61. Prado DML, Benatti FB, de Sa-Pinto AL, Hayashi AP, Gualano B, Pereira RMR, et al. Exercise training in childhood-onset systemic lupus erythematosus: a controlled randomized trial. Arthritis Research \& Therapy. 2013;15(2).

62. Omori C, Prado DML, Gualano B, Sallum AME, Sa-Pinto AL, Roschel H, et al. Responsiveness to exercise training in juvenile dermatomyositis: a twin case study. Bmc Musculoskeletal Disorders. 2010;11.

63. Nishime EO, Cole CR, Blackstone EH, Pashkow FJ, Lauer MS. Heart rate recovery and treadmill exercise score as predictors of mortality in patients referred for exercise ECG. JAMA. 2000;284(11):1392-8.

64. Cole CR, Blackstone EH, Pashkow FJ, Snader CE, Lauer MS. Heart-rate recovery immediately after exercise as a predictor of mortality. $N$ Engl $J$ Med. 1999;341(18):1351-7.

65. Ting TV, Hashkes PJ, Schikler K, Desai AM, Spalding S, Kashikar-Zuck S. The role of benign joint hypermobility in the pain experience in Juvenile Fibromyalgia: an observational study. Pediatric Rheumatology. 2012;10:6. doi: 10.1186/1546-0096-10-16.

66. Gazit Y, Nahir AM, Grahame R, Jacob G. Dysautonomia in the joint hypermobility syndrome. American Journal of Medicine. 2003;115(1):33-40. doi: 10.1016/s0002-9343(03)00235-3. 
67. Taşcılar ME, Yokuşoğlu M, Boyraz M, Baysan O, Koz C, Dundaroz R. Cardiac autonomic functions in obese children. J Clin Res Pediatr Endocrinol. $2011 ; 3(2): 60-64$.

68. Liao et al. Childhood Obesity and autonomic dysfunction: Risk for cardiac morbidity and mortality. Curr Treat Options Cardiovasc Med. 2014 October; 16(10): 342. 